\title{
Influence of the Solar and Density Perturbations on the Neutrino Parameters
}

\author{
N. Reggiani, \\ Centro de Ciências Exatas, Ambientais e de Tecnologias, Pontifícia Universidade Católica de Campinas, \\ Caixa Postal 317, 13020-970 Campinas, SP, Brasil
}

\author{
M. M. Guzzo, and P. C. de Holanda \\ Instituto de Física Gleb Wataghin, Universidade Estadual de Campinas - UNICAMP, \\ 13083-970, Campinas, São Paulo, Brasil
}

Received on 2 February, 2004

\begin{abstract}
There are reasons to believe that the solar matter density fluctuates around an equilibrium profile. One of these reasons is a resonance between the Alfvén waves and the g-modes inside the Sun that creates spikes in the density profile. The neutrinos are created in the solar core and passing through these spikes feel them as a noisy perturbation, whose correlation length is given by the distance between the spikes. When we consider these perturbations on the density profile, the values of the neutrino parameters necessary to obtain a solution to the solar neutrino problem are affected. In particular, in the present work, we show that the values of the parameters of mass and mixing angle that satisfy both the Large Mixing Angle solution to the solar neutrinos and the data from KamLAND - that observes neutrinos created in earth nuclear reactors - are shifted in the direction of lower values as the amplitude of the density noise increases. This means that, depending on the new data of KamLAND and other detectors, it can be necessary to invoke random perturbations in the Sun to recover compatibility with solar neutrino observations. In this case, the neutrino observations will be used as a real probe of the solar interior, giving information of the density profile in the central part of the Sun, which can not be observed directly.
\end{abstract}

\section{Introduction}

After the release of KamLAND data [1], it is accepted that the solution to the solar neutrino problem involves the Large Mixing Angle (LMA) realization of the MSW mechanism $[2,3]$. This experiment detected a suppression of a anti-neutrino $\bar{\nu}_{e}$ flux that were created in several nuclear reactors placed at different distances from the Kamiokande site. Interpreting this suppression as oscillation between the $\bar{\nu}_{e}$ and anti-neutrino of another flavor, the analysis of KamLAND data leads to a region for the neutrino parameters fully compatible with the LMA one [4]. Besides, KamLAND result discards all other neutrino parameter regions based on mass induced oscillations [5], such as other possible mechanism for solving the solar neutrino discrepancy, including non-standard neutrino interactions [6-8], resonant spin-flavor precession (RSFP) in solar magnetic field [9-12] and violation of the equivalence principle [1316]. The best fit values of the relevant neutrino parameters that explains both the solar neutrino and KamLAND data are $\Delta m^{2}=7.1 \times 10^{-5} \mathrm{eV}^{2}$ and $\sin ^{2} 2 \theta=0.39$ with a boron neutrino flux normalization of $f_{B}=1.04$ [4].

Besides, when we consider also the solution to the atmospheric neutrino anomaly in terms of mass induced oscillations [17] between the second and third neutrino families, and the limits in the mixing angle $\theta_{13}$ given by the CHOOZ experiment [18], we conclude that the oscillations of solar neutrinos is in fact induced only by one mass scale $\Delta m_{21}^{2}=m_{2}^{2}-m_{1}^{2}$.

But although all the mentioned mechanisms can not be the main one responsible for the solar neutrino deficit, it is interesting to explore if they can play a role as a subdominant effect in solar neutrino flavor conversion [19, 20]. Recent analysis also indicated that the solar neutrino flux detected by Super-Kamiokande is variable [21], fact that can not be easily accommodated in the pure LMA solution. Although there are some discussions if this variability is real or just a statistical fluctuation [22,23], it is interesting to keep in mind that such variability could indicate other mechanism besides the LMA acting on neutrino conversions in Sun.

With all this in mind, we believe that it is an important issue to determine if we have, as the conversion mechanism of solar neutrino, the "pure" LMA solution or if we can identify through the solar neutrino data some other mechanism acting in neutrino flavor conversion. In this context, we study in this paper the effects in the solar neutrino conversion of random solar matter density perturbations. Since the interaction of the neutrinos with solar matter is crucial to give the conversion profile in the LMA solution, possible matter fluctuations could generate fluctuations in the flavor content of solar neutrino flux. We found that random fluctuations of order of $5 \%$ in matter density can substantially affect the LMA region. 


\section{Random density perturbations in solar matter}

There are reasons to believe that the solar matter density fluctuates around an equilibrium profile. Indeed, in the hydro-dynamical approximation, density perturbations can be induced by temperature fluctuations due to convection of matter between layers with different temperatures. Considering a Boltzmann distribution for the matter density, these density fluctuations are found to be around 5\% [24]. Another estimation of the level of density perturbations in the solar interior can be given considering the continuity equation up to first order in density and velocity perturbations and the p-modes observations. This analysis leads to a value of density fluctuation around $0.3 \%$ [25]. The mechanism that might produce such density fluctuations can also be associated with modes excited by turbulent stress in the convective zone [26].

Considering helioseismology, there are constraints on the density fluctuations which would make it very unlikely that such fluctuations could lead to observational effects in solar neutrinos [27]. For the p-waves, the observed wave amplitude is too small in the solar radiative zone to affect neutrino evolution. And for the g-waves, which can have a sizeable amplitude in the solar radiative zone, the wavelength is much larger than the neutrino oscillation length, and again no effect in neutrino propagation is expected.

But recently a new mechanism [28, 29] has been proposed to generate density fluctuations with a large enough amplitude and a short enough wavelength to affect the solar neutrino oscillation. In this mechanism the shape of the g-waves can be significantly modified by a level crossing between Alfvén waves associated with a magnetic field in solar radiative zone and the g-modes. As the g-modes occur within the solar radiative zone, these resonance creates spikes at specific radii within the Sun. It is not expected that these resonances alter the helioseismic analyses because as they occur deep inside the Sun, they do not affect substantially the observed p-modes.

Before KamLAND results, it has been argued [30] that if a variability would be detected in solar neutrino flux, this could only be generated by fluctuations in solar magnetic field with the same frequency, indicating that the RSFP mechanism were acting in the solar neutrino evolution. Since the LMA region has now been established as the solution to the solar neutrino problem, discarding the RSFP mechanism as the responsible for neutrino conversion, the claimed link between solar magnetic field oscillations and solar neutrino flux variability should take a more indirect way, as for instance, the resonance between g-modes and Aflvén waves.

This resonance depends on the density profile and on the solar magnetic field, and as mentioned in Ref. [29], for a magnetic field of order of $10 \mathrm{kG}$ the spacing between the spikes is around $100 \mathrm{~km}$. The resulting wave form depends on details of the magnetic field in the solar radiative zone. For instance, if we have a vanishing radial magnetic field ( $B_{r}=0$ in polar coordinates), but with non-zero theta component $B_{\theta}$, the resonance would arise in all directions from the Sun center. This may trap the g-waves inside the resonance and, although having a strong effect in neutrino evolu- tion, can make them even more difficult to observe. If such field has a dipole form, the conditions needed to the presence of such spikes can only be realized in solar equator. As a result, only solar neutrinos detected in Earth in some periods of the year (December and June), when such neutrinos cross the solar equator, would feel those density fluctuations, and a possible seasonal variation of electron neutrino detection could arise [29].

In this paper we consider the case in which the solar matter density fluctuates by a random noise added to an average value. This is a reasonable case, considering that in the lower frequency part of the Fourier spectrum, the p-modes resembles that of noise [24]. Also, considering the resonance of g-modes with Alfvén waves, the superposition of several different modes results in a series of relatively sharp spikes in the radial density profile. The neutrino passing through these spikes feel them as a noisy perturbation whose correlation length is the spacing between the density spikes [28].

\section{Neutrino Evolution}

As stated in section 1, we will consider that conversions involves only one mass scale, and can be well described by the following $2 \times 2$ hamiltonian:

$$
i \frac{d}{d r}\left(\begin{array}{c}
\nu_{e} \\
\nu_{y}
\end{array}\right)=\left(\begin{array}{cc}
H_{e} & H_{e y} \\
H_{e y} & H_{y}
\end{array}\right)\left(\begin{array}{l}
\nu_{e} \\
\nu_{y}
\end{array}\right)
$$

where

$$
\begin{array}{r}
H_{e}=2\left[A_{e y}(t)+\delta A_{e y}\right], \\
H_{y}=0, H_{e y} \approx \frac{\Delta m^{2}}{4 E} \sin 2 \theta, \\
A_{e y}(t)=\frac{1}{2}\left[V_{e y}(t)-\frac{\Delta m^{2}}{4 E} \cos 2 \theta\right], \\
\delta A_{e y}(t) \approx \frac{1}{2} V_{e y}(t) \xi .
\end{array}
$$

Here $E$ is the neutrino energy, $\theta$ is the neutrino mixing angle in vacuum, $\Delta m^{2}$ is the neutrino squared mass difference and the matter potential for active-active neutrino conversion reads

$$
V_{e y}(t)=\frac{\sqrt{2} G_{F}}{m_{p}} \rho(t)\left(1-Y_{n}\right),
$$

where $V_{e y}$ is the potential, $G_{F}$ is the Fermi constant, $\rho$ is the matter density, $m_{p}$ is the nucleon mass and $Y_{n}$ is the neutron number per nucleon. $\xi$ is the fractional perturbation of the matter potential.

To calculate the effect of the random perturbation in matter potential, we follow the method presented in [31], where the evolution equation can be rewritten as the following:

$$
\begin{aligned}
\dot{P}(t) & =2 H_{e y} I(t) \\
\dot{R}(t) & =-H_{e y} I(t) \\
\dot{I}(t) & =H_{e}(t) R(t)-H_{e y}(2 P(t)-1)
\end{aligned}
$$


where $P(t)=\left|\nu_{e}\right|^{2}, R(t)=\operatorname{Re}\left(\nu_{y}^{*} \nu_{e}\right)$ and $I(t)=$ $\operatorname{Im}\left(\nu_{y}^{*} \nu_{e}\right)$.

Assuming that after a typical distance $L(t)$, called the correlation length of the perturbation, the density fluctuations are completely spatially uncorrelated, we can average equations (7) over one correlation length. The randomness of density fluctuations are implemented assuming that:

$$
<\delta A_{e y}(t)>=\frac{\int_{t}^{t+L(t)} A_{e y}(t) d t}{L(t)}=0
$$

and

$$
<\delta A_{e y}^{2}(t)>=\frac{1}{4} V_{e y}(t)<\xi^{2}(t)>\quad .
$$

Generalizing the above relations to higher order, we have:

$$
\begin{aligned}
<\delta A_{e y}^{2 n+1}> & =0 \\
<\delta A_{e y}(t) \delta A_{e y}\left(t_{1}\right)> & =2 k(t) \delta\left(t-t_{1}\right)
\end{aligned}
$$

where the quantity $k(t)$ is defined by

$$
k(t)=<\delta A_{e y}^{2}(t)>L(t)=\frac{1}{2} V_{e y}^{2}(t)<\xi^{2}>L(t) .
$$

Using these relations, and after some calculations, we can write the averaged products:

$$
\begin{aligned}
& <\delta A_{e y}(t) R(t)>=-k(t)<I(t)> \\
& <\delta A_{e y}(t) I(t)>=k(t)<I(t)>,
\end{aligned}
$$

and replacing in eq. (7), we can write:

$$
\begin{aligned}
<\dot{P}(t)>= & 2 H_{e y}<I(t)> \\
<\dot{R}(t)>= & -2 A_{e y}(t)<I(t)>-k(t)<R(t)> \\
<\dot{I}(t)>= & 2 A_{e y}(t)<R(t)>-2 k(t)<I(t)> \\
& -H_{e y}(2<P(t)>-1) .
\end{aligned}
$$

This approximation holds if the characteristic neutrino matter oscillation length is much bigger than the correlation length, so $L(t)$ must obey the following relations:

$$
l_{\text {free }}<<L(t)<<\lambda_{m}(t),
$$

where $l_{\text {free }}$ is the mean free path of the electrons in the Sun and $\lambda_{m}(t)$ is the characteristic neutrino matter oscillation length.

In order to guarantee that this condition is satisfied in the whole trajectory of the neutrino inside the sun, we assume that

$$
L(t)=0.1 \lambda_{m}(t)=0.1 \frac{2 \pi}{\omega(t)},
$$

where $\omega(t)$ is the frequency of the MSW effect, given by

$$
\omega^{2}(t)=4\left(A_{e y}^{2}(t)+H_{e y}^{2}\right) .
$$

We use eq. (13) to solve the neutrino evolution, and to calculate its survival probability. In Fig. 1 we show the survival probability for 4 values of the parameter $\xi, 0,2 \%, 4 \%$ and $8 \%$, in function of $\Delta m^{2} / 4 E$. We can see that for high values of $\Delta m^{2} / 4 E$ (low values of energy), when the neutrino does not feel a resonance in the Sun and the survival probability is the vacuum one, the increase of random fluctuations in solar density does not change the survival probability. For low values of $\Delta m^{2} / 4 E$ (high values of energy), the resonance is placed in the outer part of the Sun, and again, the neutrino survival probability is not affected by fluctuations in the density. So the strongest effect occurs for medium values of energy, in the transition between the vacuum regime and the resonant adiabatic conversion.

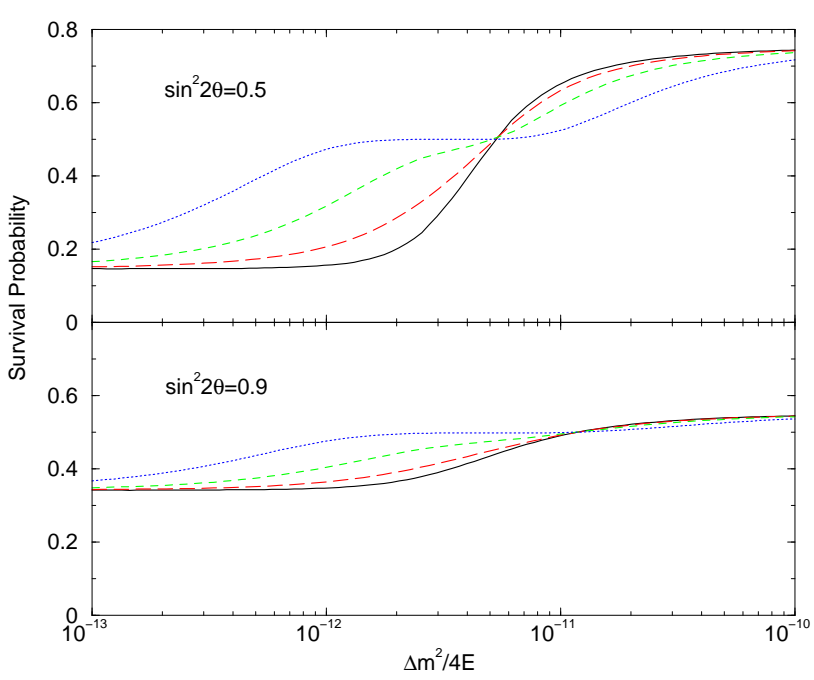

Figure 1. Neutrino survival probability for two values of the mixing angle, and several values of the perturbation amplitude, $\xi=0 \%$ (solid line), $\xi=2 \%$ (long dashed line), $\xi=4 \%$ (dashed line) and $\xi=8 \%$ (dotted line).

Other interesting feature we can notice in Fig. 1 is that the effect of random fluctuations is to bring the survival probability closer to 0.5 .

\section{KamLAND and Solar Neutrino data}

In this section we will study the effect of the solar density random fluctuations in the allowed regions in the neutrino parameter that results from the statistical analysis of solar neutrino data.

In this analysis we use the following data set:

- 3 total rates: (i) the $A r$-production rate, $Q_{A r}$, from Homestake [32], (ii) the $G e$-production rate, $Q_{G e}$, from SAGE [33] and (iii) the combined $G e$-production rate from GALLEX [34] and GNO [35];

- 44 data points from the zenith-spectra measured by Super-Kamiokande during 1496 days of operation $[36,37]$;

- 34 day-night spectral points from SNO [38];

- 3 fluxes from the SNO salt phase [39] measured by the CC-, NC and ES- reactions. We treat correlations of these fluxes following prescription in [40]. 
Altogether the solar neutrino experiments provide us with 84 data points. All the solar neutrino fluxes are taken according to SSM BP2000 [41]. This analysis is similar to the one done in [5], with the difference that in this reference the boron neutrino flux is taken as a free parameter, where here we take it according to [41].

Regarding KamLAND experiment [1], we analyzed their 13 spectrum points using the Poisson statistics, through the following $\chi^{2}$ :

$$
\chi_{K L}^{2} \equiv \sum_{i=1,13} 2\left[N_{i}^{t h}-N_{i}^{o b s}+N_{i}^{o b s} \ln \left(\frac{N_{i}^{o b s}}{N_{i}^{t h}}\right)\right]
$$

where the $\ln$ term is absent in the 5 last bins with no events. All together we have $81+13=94$ data points, with 2 parameters to fit, $\Delta m^{2}$ and $\tan ^{2} \theta$, resulting in $94-2=92$ degrees of freedom.

In absence of random fluctuations, the best fit point of our analysis lies in:

$$
\Delta m^{2}=7.2 \times 10^{-5} \mathrm{eV}^{2} \quad, \quad \tan ^{2} \theta=0.45
$$

resulting in $\chi^{2}=71.1$

In left panel of Fig. 2 we present the allowed regions in neutrino parameter space that follows from our analysis. The straight line corresponds to the allowed region with $95 \%$ C.L., with no fluctuation, when only the solar neutrino data is taken into account. point:

Our KamLAND analysis results in the following best fit

$$
\Delta m^{2}=7.24 \cdot 10^{-5} \mathrm{eV}^{2}, \quad \tan ^{2} \theta=0.52 .
$$

The filled areas represent the allowed regions that comes from the KamLAND analysis, also with 95\% C.L.

The concordance between the KamLAND and the solar neutrino data analysis is excellent. The allowed regions overlap, creating two island in the parameter space, that is called the high-LMA and low-LMA, as can be seen in Fig. 2.

In presence of the random perturbations, the allowed regions are displaced to smaller mixing angles and $\Delta m^{2}$. This can be understood by looking at Fig. 1. By increasing the size of random fluctuations, the survival probability approaches the value of 0.5 , which can be mimicked by an increase of mixing angle. Also, the distortion of spectrum that characterizes transition between resonant and non-resonant conversion is pushed to smaller values of $\Delta m^{2} / 4 E$, which reflects in a displacement of allowed regions to smaller values of $\Delta m^{2}$.

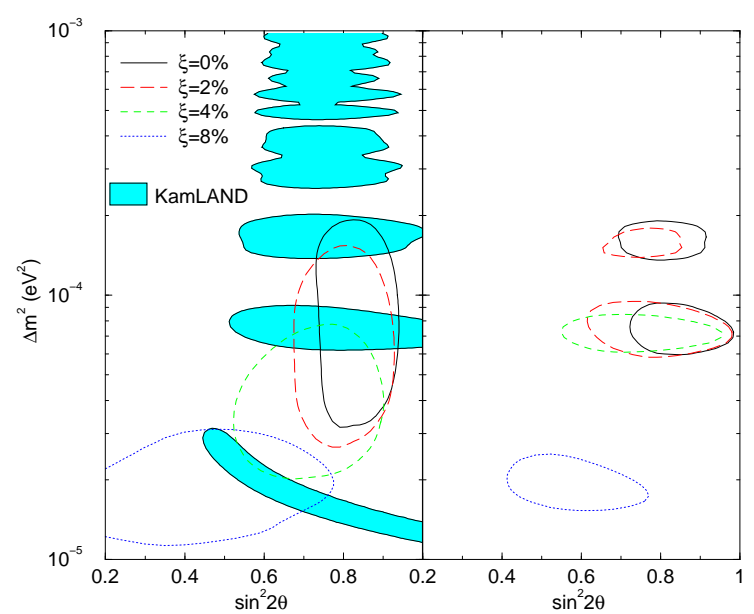

Figure 2. LMA region for different values of the perturbation amplitude, at 95\% C.L. for several values of the perturbation amplitude, $\xi=0 \%$ (solid line), for $\xi=2 \%$ (long dashed line), $\xi=4 \%$ (dashed line) and $\xi=8 \%$ (dotted line). We also present the allowed region for KamLAND spectral data, for the same C.L.. In the right-handed side of the figure, the combined analysis of both solar neutrino and KamLAND observations is shown.

Since KamLAND experiment is insensitive to fluctuations in solar density, in the combined analysis the KamLAND data prevents the allowed regions to displace in $\Delta m^{2}$. But for high enough values of the size of random fluctuations, a new region of compatibility between KamLAND and solar neutrino data appears, around $\Delta m^{2} \sim 2 \times 10^{-5}$. We call this region "very-low LMA".

In Fig. 3 it is shown $\chi^{2}$ as a function of the perturbation amplitude, minimized in $\Delta m^{2}$ and $\sin ^{2} 2 \theta$. We can see that even for high values of the perturbation amplitude we still can have a viable solution. We notice that even in a noisy scenario the compatibility of solar neutrino and KamLAND results is still good. In fact, although the absolute best fit of the analysis lies on the non-noise picture where $\xi=0$, we observe that $\left(\chi^{2}-\chi_{\min }^{2}\right)<4$ for $5 \%<\xi<8 \%$, showing a new scenario of compatibility.

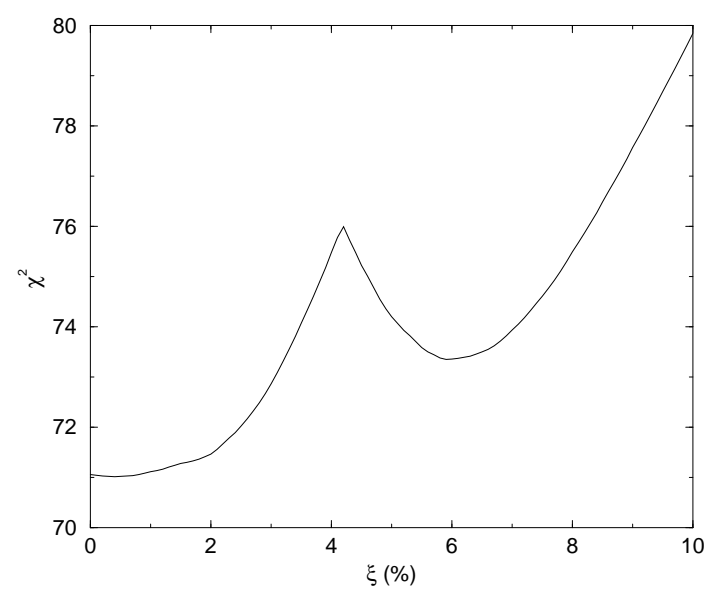

Figure 3. $\chi^{2}$ as a function of $\xi$, the perturbation amplitude, where we minimize in neutrino parameters. Here the number of degrees of freedom is 91 . 


\section{References}

[1] KamLAND collaboration, K. Eguchi et al., Phys. Rev. Lett. 90, 021802 (2003).

[2] L. Wolfenstein, Phys. Rev. D17, 2369 (1978).

[3] S.P. Mikheyev and A. Yu. Smirnov, Yad. Fiz. 42, 1441 (1985) [Sov. J. Nucl. Phys. 42, 913 (1985)], Nuovo Cimento C9, 17 (1986); S.P. Mikheyev and A. Yu. Smirnov, ZHTEF 91 (1986) [Sov. Phys. JETP 64, 4 (1986)].

[4] P. C. de Holanda and A. Yu. Smirnov, hep-ph/0309299, accepted to be published at Astropart. Phys.

[5] P. C. de Holanda and A. Yu. Smirnov, Journ. of Cosm. and Astropart. Phys. 02, 001 (2003).

[6] M.M. Guzzo, A. Masiero and S. T. Petcov, Phys. Lett. B 260, 154 (1991).

[7] E. Roulet, Phys. Rev. D 44, 935 (1991).

[8] S. Bergmann, M.M. Guzzo, P.C. de Holanda, P. Krastev and H. Nunokawa, Phys. Rev. D 62, 073001 (2000).

[9] J. Schechter and J. W. F. Valle, Phys. Rev. 24, 1883 (1981); ibid. 25, 283 (1982).

[10] C. S. Lim and W. J. Marciano, Phys. Rev. 37, 1368 (1988).

[11] E. Kh. Akhmedov, Sov. J. Nucl. Phys. 48, 382 (1988); Phys. Lett. B 213, 64 (1988)

[12] M. M. Guzzo and H. Nunokawa, Astropart. Phys. 12, 87 (1999).

[13] M. Gasperini, Phys. Rev. D 38, 2635 (1988); ibid. 39, 3606 (1989).

[14] A. M. Gago, H. Nunokawa and R. Zukanovich Funchal, Phys. Rev. Lett. 84, 4035 (2000); Nucl. Phys. (Proc. Suppl.) B 100 , 68 (2001).

[15] A. Halprin and C. N. Leung, Phys. Rev. Lett. 67, 1833 (1991).

[16] S. W. Mansour and T. K. Kuo, Phys. Rev. D 60, 097301 (1999); A. Raychaudhuri and A. Sil, hep-ph/0107022.

[17] Y. Fukuda et. al. [Super-Kamiokande Collaboration], Phys. Rev. Lett. 81, 1562 (1998).

[18] CHOOZ Collaboration, M. Apollonio et al., Phys.Lett. B466, 415 (1999); Eur. Phys. J. , C 27, 331 (2003).

[19] P. C. de Holanda and A. Yu. Smirnov, hep-ph/0307266.

[20] J. Barranco, O.G. Miranda, T.I. Rashba, V.B. Semikoz, J.W.F. Valle, Phys. Rev. D 66093009 (2002); O. G. Mirana, T. I. Rashba, A. I. Rez, J. W. F. Valle, hep-ph/0311014.

[21] P.A. Sturrock, Astrophys. J. 594, 1102 (2003).

[22] J. Yoo et al. [Super-Kamiokande collaboration], Phys. Rev. D 68092002 (2003);
[23] P. A. Sturrock, hep-ph/0309239; D.O. Caldwell, P.A. Sturrock, hep-ph/0309191.

[24] H. Nunokawa, A. Rossi, V. B. Semikoz, J. W. F. Valle, Nucl. Phys. B 472, 495 (1996).

[25] S. Turck-Chiéze and I. Lopes, Ap. J 408 (1993) 346; S. Turck-Chiéze et al., Phys. Rep. 230 (1993) 57.

[26] P. Kumar, E. J. Quataert and J. N Bahcall, Astrophys. Journ. 458, L83 (1996).

[27] P. Bamert, C. P. Burgess and D. Michaud, Nucl. Phys. B 513, 319 (1998).

[28] C. Burgess et al., hep-ph/0209094 v1.

[29] C. P. Burgess, N. S. Dzhalilov, T. I. Rashba, V. B. Semikoz and J. W. F. Valle, M.N.R.A.S. (to appear), astro-ph/0304462.

[30] N. Reggiani, M. M. Guzzo and P.C. de Holanda, Braz. J. Phys. 33, 767 (2003). M. M. Guzzo, P. C. de Holanda, N. Reggiani, Eur.Phys.J.C 25, 459 (2002); N. Reggiani, M. M. Guzzo, J.H. Colonia and P. C. de Holanda, Eur. Phys. J. C 12, 263 (2000);

[31] H. Nunokawa, A. Rossi, V. B. Semikoz, J. W. F. Valle, hep$\mathrm{ph} / 9602307$.

[32] B. T. Cleveland et al., Astroph. J. 496, 505 (1998).

[33] SAGE collaboration, J.N. Abdurashitov et al. Zh. Eksp. Teor. Fiz. 122, 211 (2002) [J. Exp. Theor. Phys. 95, 181 (2002)], astro-ph/0204245; V. N. Gavrin, Talk given at the VIIIth International conference on Topics in Astroparticle and Underground Physics (TAUP 03), Seattle, Sept. 5 - 9, 2003.

[34] GALLEX collaboration, W. Hampel et al., Phys. Lett. B 447, 127 (1999).

[35] GNO Collaboration, E. Belotti, Talk given at the VIIIth International conference on Topics in Astroparticle and Underground Physics (TAUP 03), Seattle, Sept. 5 - 9, 2003.

[36] Super-Kamiokande collaboration, S. Fukuda et al., Phys. Rev. Lett. 86, 5651 (2001); Phys. Rev. Lett. 86, 5656 (2001), Phys. Lett. B 539, 179 (2002).

[37] Super-Kamiokande collaboration, M. B. Smy et al., hepex/0309011

[38] SNO collaboration, Q. R. Ahmad et al.; ibidem 87, 071301 (2001); ibidem 89, 011301 (2002); ibidem 89, 011302 (2002).

[39] SNO collaboration (Q. R. Ahmad et al.), nucl-ex/0309004.

[40] "HOWTO use the SNO salt flux results", website: www.sno.phy.queensu.ca .

[41] J. N. Bahcall, M.H. Pinsonneault and S. Basu, Astrophys. J. 555, 990 (2001). 